\title{
Materia prima para la industria cerámica de Norte de Santander. II. Evaluación del comportamiento térmico y su incidencia en las propiedades tecnológicas
}

\section{Raw material for the ceramic industry in Norte de Santander. II. Evaluation of thermal behavior and its impact on the technological properties}

\section{Matéria prima para a indústria cerâmica de Norte de Santander. II. Evacuação do comportamento térmico e sua incidência nas propriedades tecnológicas}

\author{
Leonardo Cely-Illera ${ }^{1}$, Rafael Bolívar-León ${ }^{2}$
}

Forma de citar: L. Cely, R. Bolívar, "Materia prima para la industria cerámica de Norte de Santander. II. Evaluación del comportamiento térmico y su incidencia en las propiedades tecnológicas”, Respuestas, vol. 20, no. 1, pp. 84-94, 2015 .

Recibido:

3 de Junio 2014

Aceptado:

10 de Noviembre 2014

\section{Resumen}

Antecedentes: Este trabajo constituye la continuación de uno previo; en la primera parte de este trabajo se estudiaron las características mineralógicas, químicas y físicas de una arcilla del área metropolitana de San José de Cúcuta. Objetivos: En este trabajo se realizaron análisis térmicos de termogravimetria (ATG) y térmico-diferencial (ATD) para desarrollar una curva optima de cocción. Metodología: Se sinterizaron muestras extruidas y se realizó la caracterización tecnológica del mismo, ensayos de absorción de agua, resistencia mecánica y abrasión profunda, se desarrollaron. Además quemas en un horno industrial con el fin de realizar un comparativo de la curva propuesta y una curva de cocción real. Resultado: Se pudo comprobar que al usar la curva propuesta en la investigación y comparando con los resultados arrojados a nivel industrial, no solo se sacó el mejor provecho de este recurso, sino se determinó que la materia prima mejora considerablemente sus características tecnológicas, incrementando el tráfico del producto. Conclusiones: Esto significaría la posibilidad de mejores productos, generando competitividad en cualquier tipo de mercado no sólo a nivel nacional sino internacional.

Palabras Clave:Conformado, Desgaste, Microestructura, Propiedades Mecánicas, Sinterización.
Abstract
Background: This work is a continuation of previous one; in the first part of this work mineralogical, chemical and physical characteristics of a clay of metropolitan San José de Cúcuta was studied. Objective: In this work, thermal-differential (ATG) and Thermogravimetry analysis (ATD) were performed in order to develop an optimal firing curve. Methods: Sintering extruded samples and technological characterization of the same, testing water absorption, mechanical strength, deep abrasion and burning 
in an industrial furnace were performed in order to develop a comparative of the proposal curve and the firing real curve. Results: It was proved that using the curve given in the research and comparing it with the results obtained at industrial level, not only the most of this resource was removed, but also it was determined that raw material greatly enhances its technological characteristics, increasing traffic product. Conclusions: Which would mean the possibility of better products, generating competitive in any market not only nationally but internationally.

Keywords: Shaping, Wear, Microstructure, Mechanical Properties, Sintering.

\section{Resumo}

Antecedentes: Este trabalho constitui a continuação de um anterior; na primeira parte deste trabalho se estudaram as características mineralógicas, químicas e físicas de uma argila da área metropolitana de San José de Cúcuta. Objetivos: Neste trabalho se realizaram análises térmicos de termogravimétrica (ATG) e térmico-diferencial (ATD) para desenvolver uma curva ótima de cocção. Metodologia: Sinterizaram-se amostras estruídas e realizou-se a caracterização tecnológica das mesmas, ensaios de absorção de agua, resistência mecânica e abrasão profunda, também foram desenvolvidos. Além disso, foram realizadas queimas num forno industrial com o fim de realizar um comparativo entre a curva proposta e a curva de cocção real. Resultado: Conseguiu-se comprovar que ao usar a curva proposta na pesquisa e comparando com os resultados obtidos a nível industrial, não só se tirou o melhor proveito deste recurso, senão que se determinou que a matéria prima melhora consideravelmente suas características tecnológicas, incrementando o trafego do produto. Conclusões: Isto significaria a possibilidade de melhores produtos, gerando competitividade em qualquer tipo de mercado não só a nível nacional senão internacional.

Palavras-Chave: Conformado, Desgaste, Microestrutura, Propriedades Mecânicas, Sinterização.

\section{Introducción}

Un producto cerámico (Teja, ladrillo, baldosa, etc.) puede mantener sus propiedades técnicas y estéticas durante un largo período de tiempo, a no ser que sea utilizado en unas determinadas condiciones que ocasionaría un elevado deterioro, lo que se traduce en una disminución de su vida útil. Por esta razón el punto de llegada de un proceso es proporcionar a un material una estructura resistente a cualquier desgate o deterioro generado por estos fenómenos ambientales (mecánicos o físicos), y de esta manera mejorar las propiedades y lograr que estas se mantengan inalterables durante un lapso establecido [1].

Las propiedades tecnológicas tienen una gran importancia en un producto, la falta de estas conduce a la aparición de bajas y por consiguiente a un alto costos de producción que se ve reflejado al final del proceso [2]; estas propiedades dependen inicialmente de la composición química, física o mineralógica de las materias primas que hacen parte en una pasta, pero pueden ser modificadas en el momento que el material es sometido a la acción del calor. Por este motivo es importante trabajar una curva de cocción que ayude a mejorar la propiedades de un producto cerámico, esto se logra controlando todo el proceso de cocción y evaluando la incidencia de este en las propiedades finales del mismo [3]; y de esta manera lograr mejores calidades técnicas en un menor tiempo de trabajo [4].

Enero - Junio 2015

PP: 84-94

Partiendo de estas observaciones la presente investigación se dirigió hacia el estudio de 
Materia prima para la industria cerámica de Norte de Santander. II. Evaluación del comportamiento térmico y su incidencia en las propiedades tecnológicas una curva óptima, que mejoro las propiedades tecnológicas de los productos fabricados con una arcilla de la región de Norte de Santander. Propiedades como resistencia mecánica a la flexión y a la abrasión, porosidades y comportamientos físico-cerámicos, entre otras, fueron estudiadas sacando el mejor provecho de este recurso y obteniendo resultados que generaron valor agregado $\mathrm{y}$ una posible competitividad en el mercado internacional.

\section{Materiales y métodos}

\subsection{Materiales}

La arcilla investigada proviene de la región de El Zulia, municipio de Norte de Santander, pertenece a la formación Guayabo y es explotada por empresas de la región. La arcilla es de color rojizo y de textura plástica [19].

\subsubsection{Equipos empleados}

Se usó una termobalanza Q600 de alta sensibilidad marca TA Instruments, una estufa de acero Inoxidable con ventilación natural entre un rango de $0^{\circ} \mathrm{C}$ hasta $300^{\circ} \mathrm{C}$, un horno mufla de 64 litros de volumen, una estufa de calefacción, una prensa de presión (Crometro) y un abrasimetro CAP/3 todos marca Gabrielli, un calibrador digital marca Mitutoyo y sensibilidad $0,01 \mathrm{~mm}$ y una balanza digital marca OHAUS adventurer de capacidad $1500 \mathrm{~g}$ de sensibilidad 0,01g. En la Figura 1 se muestran algunos equipos empleados.

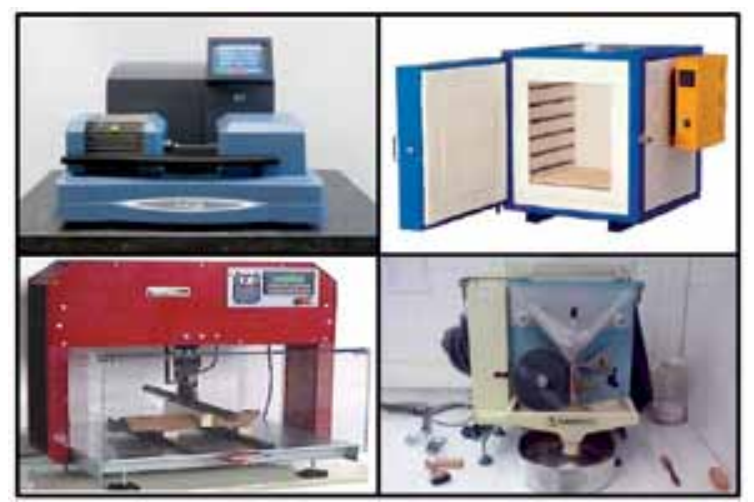

Figura 1. Equipos empleados durante lainvestigación. Fuente: Centro de Investigación de Materiales Cerámicos -UFPS- y Laboratorio de calorimetría; Universidad de Pamplona.

\subsection{Métodos}

- Análisis térmicos. Se realizaron ensayos térmico-diferencial (ATD) y análisis termo-gravimétrico (ATG) en un rango de temperatura entre temperatura ambiente y $1200^{\circ} \mathrm{C}$. El ensayo se realizó en presencia de una atmosfera controlada de oxígeno y en un ciclo térmico de $5^{\circ} \mathrm{C} /$ min.

- Absorción de agua. Se determinó mediante el método de ebullición según la norma NTC 4321-3.

- Resistencia mecánica a la flexión. El estudio de esta propiedad se ha determinado mediante la norma NTC 4321-4.

- Resistencia a la abrasión profunda. El estudio de esta propiedad se ha determinado mediante la norma NTC 4321-6.

\subsubsection{Procedimiento}

Utilizando una arcilla de la formación Guyabo de la región oriental del departamento de Norte de Santander, la cual fue caracterizada y estudiada en [19], se conformaron las probetas necesarias para la investigación. Inicialmente se humectó el material, se amasó y se dejó en reposo por 12 horas para homogenizar la masa arcillosa. Se inició el proceso de conformado, este se realizó por el método de extrusión para obtener probetas rectangulares con dimensiones de $11 \mathrm{~cm} *$ $4 \mathrm{~cm} * 1 \mathrm{~cm}$, como se indica en [5]. A cada probeta conformada se le determinaron las dimensiones y masa en estado en verde (estado húmedo). Realizado este procedimiento las probetas se sometieron al proceso de secado, iniciando el proceso a temperatura ambiente por las primeras 5 horas, después en estufa de secado por un tiempo de 12 horas a una temperatura de $60^{\circ} \mathrm{C}\left( \pm 5^{\circ} \mathrm{C}\right)$ y por último aumentando gradualmente la temperatura hasta $110^{\circ} \mathrm{C}\left( \pm 5^{\circ} \mathrm{C}\right)$ por un tiempo de 24 
horas. Finalizado este proceso nuevamente se evaluaron las dimensiones y la masa en estado seco. A continuación se desarrollaron los análisis térmicos, durante este proceso se mantuvo constante la atmosfera de oxígeno, el caudal y la velocidad de calentamiento que fue de $5^{\circ} \mathrm{C} / \mathrm{min}$ y se llevó el material a una temperatura final de $1.200^{\circ} \mathrm{C}$ [6]. Terminado los análisis, se evaluó, se analizó y al final se estipulo una curva tentativa que se aplicó al material, con el objetivo de poder observar el comportamiento de las propiedades tecnologías de la materia prima. Esta curva se realizó en un horno mufla y después de la cocción, a las probetas se le determinaron las condiciones cocidas (Dimensiones y masa) y se realizaron ensayos de absorción de agua, de resistencia mecánica a la flexión y ensayos de resistencia a la abrasión profunda. Por último se analizan los resultados arrojados en esta investigación y se aportan conclusiones.

\section{Resultados y análisis}

\subsection{Estudio térmico}

En la figura 2, aparecen las curvas de análisis térmico. En general, esta muestra es característica de mezclas formadas por minerales micáceos y caoliniticos; en efecto, en esta se puede observar que la curva ATD de color verde, en el punto ( 0 ) entre la temperatura ambiente y los $50^{\circ} \mathrm{C}$, es característico de una curva tipo ii, donde ocurren procesos de desorción o secado; representa un pequeño pico endotérmico relacionado a la eliminación del agua higroscópica o humedad residual, acompañada con una pérdida de peso de $1,70 \%$. Probablemente la fase higroscópica illitica del material reabsorbió humedad del ambiente previamente al ensayo [7]. Entre los puntos $(0-\mathrm{A})$ entre los $50^{\circ} \mathrm{C}$ y los $250^{\circ} \mathrm{C}$ se aprecia una ligera estabilización en la curva, acompañado de una pérdida de masa de $3,36 \%$, producto de la evaporación del agua absorbida (Agua zeolítica), ligada por absorción en las estructuras cristalinas [5].

Entre los puntos (A - R - C) correspondiente a temperaturas de $250^{\circ} \mathrm{C}$ a $850^{\circ} \mathrm{C}$, la curva registra un descenso gradual y progresivo, alcanzando su punto más bajo sobre los $850^{\circ} \mathrm{C}$. Este efecto corresponde a la pérdida del agua de cristalización (Deshidroxilación de la arcilla), que se prolongaría hasta la

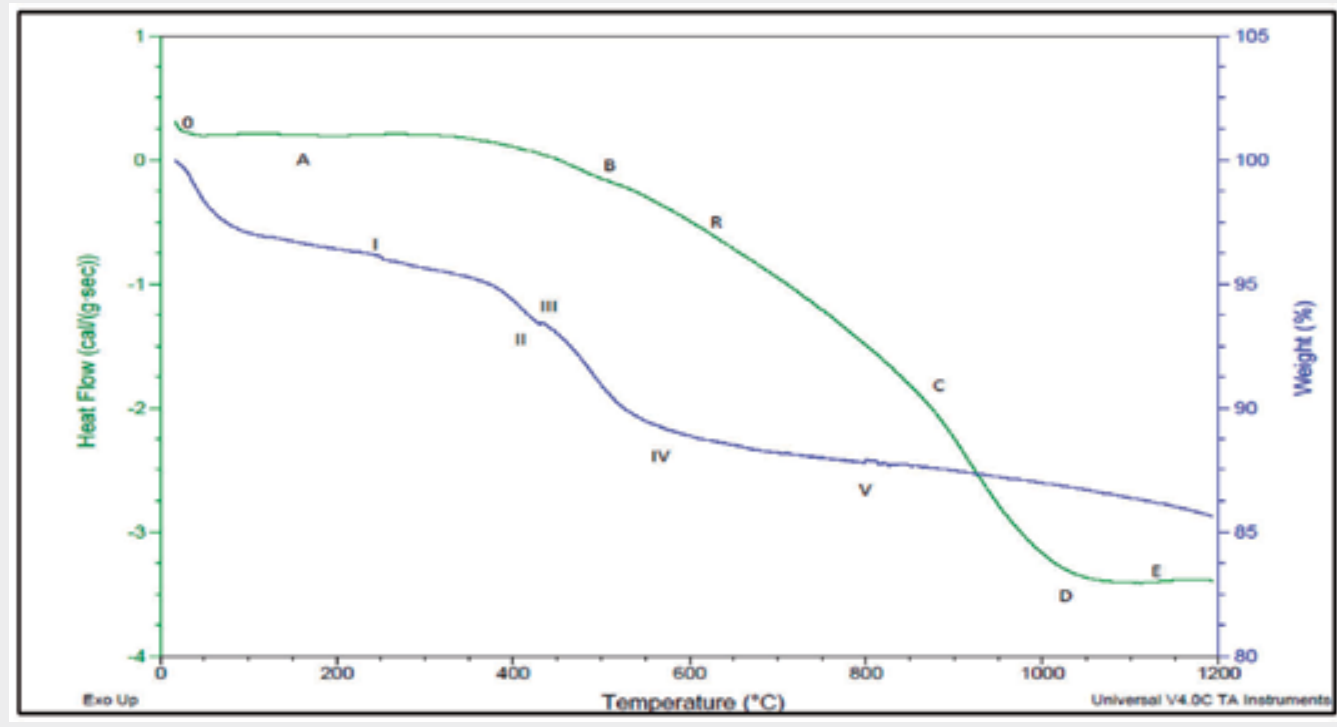

Figura 2. Curvas de análisis térmicos de la muestra que se investigó Fuente: Autores (Laboratorio de calorimetría; Universidad de Pamplona, Pamplona, Colombia). 
temperatura de fusión del material [8]. Entre los puntos (C - D), se observa un leve pico, debido a la formación de nuevas fases cristalinas constituidas por la sílice $\left(\mathrm{SiO}_{2}\right)$ de los silicatos y silicoaluminatos complejos, además se observa una variación en el punto V de la curva ATG, a pesar de que el porcentaje en peso de $\mathrm{CaO}$ y $\mathrm{MgO}$ es muy bajo como se observa en la tabla I, esto es debido a la descarbonatación de la calicita con la liberación de $\mathrm{CO}_{2}$. Finalmente entre los puntos (D - E) entre las temperaturas de $1000^{\circ} \mathrm{C}$ a los $1200^{\circ} \mathrm{C}$, se produce una reacción exotérmica que coincide con la formación de la fase cristalina conocida como mullita ( $\mathrm{La}$ presencia de mullita en un producto denota una buena cocción y constituye una garantía de calidad, además del inicio de la vitrificación de la muestra y el aumento de la resistencia mecánica de la pieza), esta fase aumenta hasta los $1.200^{\circ} \mathrm{C}$ [5], [6], [7], [9], [10], [11], [12]. Por último en la curva termo-gravimétrica de color azul, se observa en los puntos II y III una variación (Tipo iii) con una pérdida de masa $6,52 \%$, esto se debe a la disociación oxidante de los hidróxidos de hierro, además en el punto IV se observa una inflexión entre las temperaturas $500^{\circ} \mathrm{C}$ y $650^{\circ} \mathrm{C}$, se asocia a la transformación alotrópica del cuarzo $(\alpha-\beta)$, muy común en las arcillas y que ocurre a los $573^{\circ} \mathrm{C}$

Tabla I. Análisis de fluorescencia de la muestra de arcilla (FRX)

\begin{tabular}{|c||c|}
\hline \multicolumn{1}{|c||}{ ELEMENTO } & COMPOSICIÓN \\
\hline \hline FORMULA & \% en masa \\
\hline $\mathrm{SiO}_{2}$ & 64,87 \\
\hline $\mathrm{Al}_{2} \mathrm{O}_{3}$ & 20,56 \\
\hline $\mathrm{Fe}_{2} \mathrm{O}_{3}$ & 4,75 \\
\hline $\mathrm{Na}_{2} \mathrm{O}$ & 0,15 \\
\hline $\mathrm{K}_{2} \mathrm{O}$ & 1,63 \\
\hline $\mathrm{CaO}$ & 0,22 \\
\hline \hline $\mathrm{MgO}$ & 0,75 \\
\hline \hline $\mathrm{SO}_{3}$ & 0,04 \\
\hline \hline PERDIDAS POR IGNICIÓN & 0,99 \\
\hline \hline SIN DETERMINAR & 6,04 \\
\hline TOTAL & 100,00 \\
\hline RELACIÓN MOLAR $\left(\mathrm{SiO}_{2} / \mathrm{Al}_{2} \mathrm{O}_{3}\right)$ & 5,40 \\
\hline
\end{tabular}

Fuente: Autores

\subsection{Cocción del material}

La optimización del proceso de cocción debe comenzar con el establecimiento de la curva ideal de temperaturas y tiempo, que permita evitar las roturas durante el precalentamiento, cocción o enfriamiento. Estas roturas, son producidas por tensiones derivadas de las diferencias de contracción/dilatación que tienen lugar dentro de la pieza, que dependen a su vez, de los gradientes térmicos que en un momento determinado puedan existir en el material, los cuales varían en función de las reacciones endotérmicas o exotérmicas observadas en la Figura 2 [5].

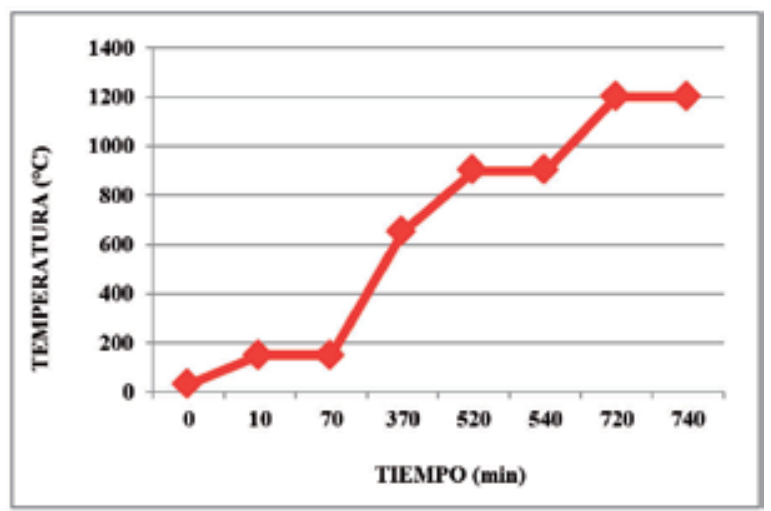

Figura 3. Curva de cocción propuesta Fuente: Autores

Por esta razón y con el análisis del termograma; en la curva propuesta (Figura 3) se planteó que durante la etapa de precalentamiento, el tiempo de aumento de la temperatura sea alrededor de 40 minutos con una permanecía de 30 minutos en la temperatura de $150^{\circ} \mathrm{C}$, con el fin de evaporar la humedad residual. En la segunda etapa se propuso aumentar la temperatura gradualmente (aproximadamente $1,67^{\circ} \mathrm{C} / \mathrm{min}$ ) durante 300 minutos hasta alcanzar los $650^{\circ} \mathrm{C}$, con el fin de descomponer por completo el material arcilloso, disociar los hidróxidos de hierro y evitar el incremento de la masa que se observó en los puntos II y III del termograma; además de disminuir la inflexión que se observa entre los $500^{\circ} \mathrm{C}$ y $\operatorname{los} 650^{\circ} \mathrm{C}$, ocasionada por el cuarzo. Entre las temperaturas de $650^{\circ} \mathrm{C}$ a $900^{\circ} \mathrm{C}$, se propuso 
que el calentamiento tenga una duración de 150 minutos y una vez alcanzado este set point se mantenga durante 20 minutos y así controlar el leve pico endotérmico entre los puntos $\mathrm{C}-\mathrm{D}$ y la variación en el punto V. Por último, se propuso un tiempo de calentamiento de 180 minutos (aproximadamente $1,67^{\circ} \mathrm{C} /$ min) para los puntos $\mathrm{D}$ - E de la curva, entre los $900^{\circ} \mathrm{C}$ a $1200^{\circ} \mathrm{C}$ o la temperatura final a la que se vaya a trabajar y una mesa de cocción de 20 minutos en la temperatura máxima para controlar el pico exotérmico desarrollado a partir de $1050^{\circ} \mathrm{C}$.

Con estos datos finalmente se propuso la curva térmica, la cual redujo las tensiones sufridas en esta etapa del proceso, se validó realizando el proceso de cocción a nivel de laboratorio usando un horno mufla (Figura 1) y se realizaron cocciones en un horno industrial tipo colmena (o de llama invertida) y con curvas usadas en ellos, esto con el fin de comparar los datos arrojados por la curva propuesta. En el horno colmena las cocciones se ejecutaron en atmósfera oxidante y reductora. Las variables de los procesos de cocción se pueden observar en la Tabla II. Se señala que el proceso de cocción en los hornos colmenas se realiza con ciclos de cocción lentos y se usa carbón natural como combustible orgánico; este proceso puede durar hasta 12 días.

Tabla II. Ciclo de cocción en los hornos industriales.

\begin{tabular}{|c|c|c|c|c|}
\hline HORNO & ATMOSFERA & CICLO & $\begin{array}{l}\text { DENOMINACIÓN EN } \\
\text { LA INVESTIGACIÓN }\end{array}$ & $\begin{array}{l}\text { TEMPERATURA } \\
\text { FINAL } \\
\left({ }^{\circ} \mathrm{C}\right)\end{array}$ \\
\hline \multirow{3}{*}{ COLMENA } & \multirow{2}{*}{ REDUCTORA } & $\begin{array}{c}\text { TIEMPO DE REDUCCIÓN } \\
1 \text { hora } 40 \text { minutos }\end{array}$ & M1 & $960-1007$ \\
\hline & & $\begin{array}{c}\text { TIEMPO DE REDUCCIÓN } \\
1 \text { hora } 30 \text { minutos }\end{array}$ & M2 & $1030-1080$ \\
\hline & OXIDANTE & LA EMPRESA NO ENTREGO DATOS & M3 & $1050-1100$ \\
\hline
\end{tabular}

Fuente: Empresas regionales

\subsection{Estudios tecnológicos}

3.3.1. Análisis físico-cerámico (Contracción lineal). Como se observa en la Figura 4, las contracciones ocurridas en el horno mufla al usar la curva de cocción propuesta con respecto a los resultados obtenidos en las diferentes atmosferas del horno colmena, son de $0.03 \%, 0.28 \%$ y $0.11 \%$ con relación a lo ocurrido en el proceso industrial de M1, M2 y M3 respectivamente; además de tener menos dispersión entre los mismos resultados. Esto se debe a que al aplicar la curva de cocción propuesta, el material alcanza completamente gresificación, ya que las fases sílicoaluminatos presentes en la muestra inician su proceso de reblandecimiento, fundiéndose y con ello, haciendo que las contracciones aumenten ligeramente pero otorgando al producto final buena dureza, compacidad e impermeabilidad y reduciendo su porosidad [13], además de evitar roturas en el material.

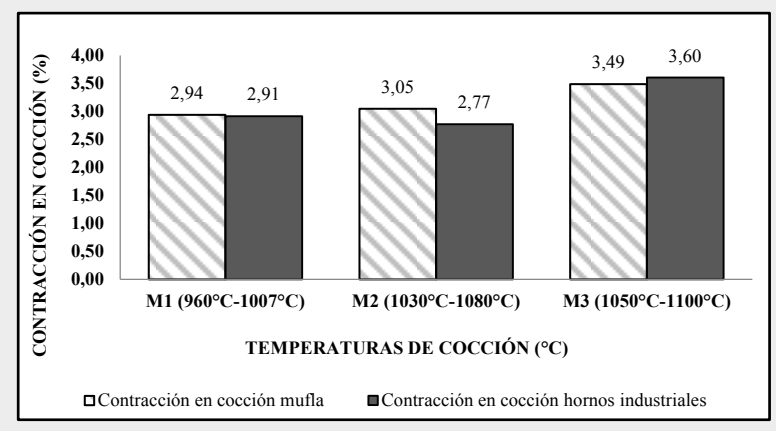

Figura 4. Contracciones lineales en cocción. Fuente: Autores

3.3.2.Absorción de agua. Como se observa en la figura 5 , los resultados arrojados en la curva propuesta para M1, M2 y M3 fueron menores, estos disminuyeron en $0,29 \%, 1,39 \%$ y $0,63 \%$ respectivamente $\mathrm{y}$ en promedio $0,77 \%$. 
Materia prima para la industria cerámica de Norte de Santander. II. Evaluación del comportamiento térmico y su incidencia en las propiedades tecnológicas

Estos valores nos indican que el material durante el proceso de cocción tuvo una mayor densificación, mejorando propiedades tecnologías como resistencias mecánicas o químicas [14]. Es decir, se logró controlar las reacciones que ocurren dentro del proceso de cocción, principalmente la deshidroxilación del mineral arcilloso y la sinterización parcial de la masa en la formación de fase vítrea entre los bordes de grano, que son generadores de porosidad [15].

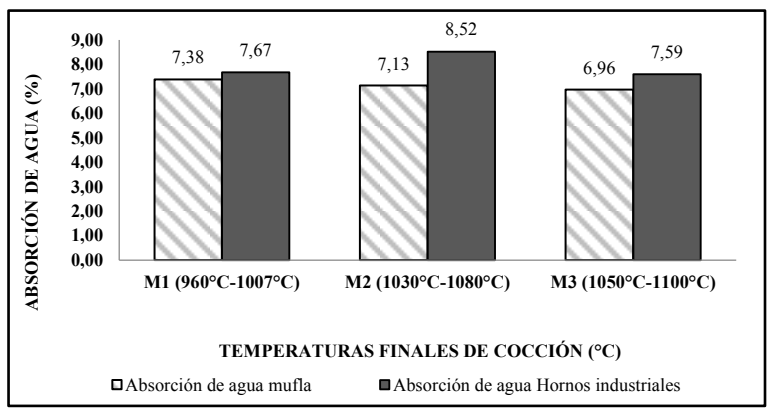

Figura 5. Absorción de agua.

Fuente: Autores

3.3.3. Resistencia mecánica a la flexión. Se detalla que en la curva propuesta el material aumento su resistencia a la flexión en $0.03 \mathrm{~N} / \mathrm{mm}^{2}, 8,87 \mathrm{~N} / \mathrm{mm}^{2}$ y $0,93 \mathrm{~N} / \mathrm{mm}^{2}$, respectivamente para $\mathrm{M} 1, \mathrm{M} 2$ y $\mathrm{M} 3$, como se observa en la Figura 6. Las variaciones para M1 y M3 son buenas, la alta variación respecto a M2 se puede explicar, en razón que, con la curva propuesta se logró un mejor control en la velocidad de calentamiento, en las reacciones durante el proceso y un mayor grado de vitrificación, esto se observa en el hecho que, aumentó su contracción lineal, se densificó mejor y disminuyó su porosidad, situación que no se logró al reducir el tiempo de reducción en el horno industrial. Cabe resaltar que los resultados de resistencia mecánica se relacionan directamente con los de absorción de agua, pues las propiedades mecánicas en los materiales cerámicos están muy influenciadas por la porosidad [15]. Los resultados para cualquiera de las temperaturas trabajadas en esta investigación, están por encima de los valores normales de resistencia mecánica en arcillas utilizadas para productos de cerámica estructural, los cuales están comprendidos entre $100 \mathrm{Kg} / \mathrm{cm}^{2}$ $\left(9,81 \mathrm{~N} / \mathrm{mm}^{2}\right)$ y $150 \mathrm{Kg} / \mathrm{cm}^{2} \quad\left(14,72 \mathrm{~N} / \mathrm{mm}^{2}\right)$ superan los umbrales normales de resistencia mecánica sino mejora los resultados arrojados industrialmente.

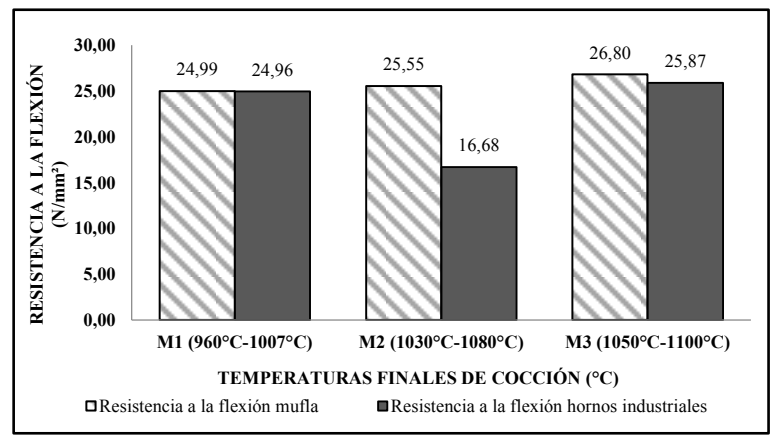

Figura 6. Resistencia mecánica a la flexión.

Fuente: Autores

3.3.4. Resistencia a la abrasión profunda. La resistencia mecánica a la abrasión profunda, permite conocer el grado de cohesión interna de un material, respecto a la agresión que se da en la aplicación de una fuerza de rozamiento externa, y al igual que la resistencia mecánica a la flexión, sirve para saber si los productos fabricados pueden cumplir con los mínimos estándares especificados por las normas nacionales e internacionales para determinados productos.

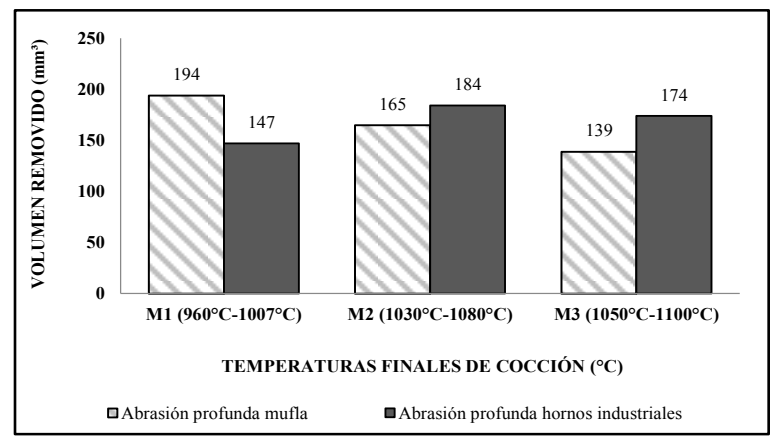

Figura 7. Abrasión profunda en baldosas no esmaltadas

Fuente: Autores

Como se observa en la Figura 7, el desgaste que sufre el material al aplicarle la curva de cocción propuesta es menor en M2 y M3, con valores de $165 \mathrm{~mm}^{3}$ y $139 \mathrm{~mm}^{3}$ respectivamente, mejorando la calidad del [16], pero con la curva propuesta no solo se 
producto, esta propiedad esta influenciadas por la porosidad de la misma manera como la resistencia mecánica a la flexión [15]. El alto desgaste que sufre el material en M1, puede ser consecuencia de las cantidades relativamente alta de $\mathrm{Al}_{2} \mathrm{O}_{3}$, y de $\mathrm{Fe}_{2} \mathrm{O}_{3}$, además de la baja cantidad de alcalinos y su estructura caolinitico-illitico, que le da al material tendencia a ser refractario, por lo cual este tipo de arcillas a temperaturas de procedimientos bajas pueden no lograr alcanzar las máximas propiedades que otro proceso a mayores temperaturas obtenidas [8].

\subsection{Discusión}

Con el fin de corroborar la eficiencia y la eficacia de la curva propuesta, se realizó un comparativo de los resultados obtenidos en los hornos industriales y las curvas de cocción comúnmente usadas en la región, con los datos arrojados por el horno mufla aplicándole la curva de cocción propuesta; se compararon los tráficos en cada una de las temperaturas y se observaron los cambios obtenidos en cada una de las características tecnológicas del material, de la siguiente manera:

3.4.1. Análisis comparativo de los resultados obtenidos entre el proceso de cocción de M1 realizado en el horno colmena con atmosfera reductora a temperatura entre $960^{\circ} \mathrm{C}-1007^{\circ} \mathrm{C}$ (1 hora y 40 minutos de reducción) y la curva de cocción propuesta en el horno mufla.

Tabla III. Tabla comparativa entre los hornos mufla y colmena con atmosfera reductora baja.

\begin{tabular}{|c|c|c|c|c|}
\hline \multirow{3}{*}{ HORNO } & NTC 4321-3 & $\begin{array}{c}\text { ANÁLISIS } \\
\text { FÍSICO-CERÁMICO } \\
\text { ASTM C326-03 } \\
\end{array}$ & NTC 4321-4 & NTC 4321-6 \\
\hline & ABSORCIÓN DE AGUA & $\begin{array}{l}\text { CONTRACCIÓN } \\
\text { COCIDA }\end{array}$ & $\begin{array}{c}\text { RESISTENCIA A } \\
\text { LA FLEXIÓN O } \\
\text { MODULO DE } \\
\text { RUPTURA }\end{array}$ & $\begin{array}{l}\text { RESISTENCIA A LA } \\
\text { ABRASIÓN PROFUNDA }\end{array}$ \\
\hline & \% & \% & $\mathbf{N} / \mathbf{m m}^{2}$ & $\mathbf{m m}^{3}$ \\
\hline MUFLA & 7,38 & 2,94 & 24,99 & 194 \\
\hline $\begin{array}{c}\text { COLMENA ATMOSFERA } \\
\text { REDUCTORA } \\
\text { BAJA (M1) }\end{array}$ & 7,67 & 2,91 & 24,96 & 146 \\
\hline
\end{tabular}

Fuente: Autores

Teniendo en cuenta que las características de resistencia a la flexión y la abrasión profunda, definen el tráfico de una baldosa según su uso por características mecánicas y realizando el comparativo con los resultados obtenidos en el proceso de cocción, se puede determinar, que el material sinterizado en el horno industrial obtiene productos de referencia a un tráfico 2 "Pavimento tránsito peatonal leve", mientras que el obtenido usando la curva de cocción propuesta en el horno mufla es de referencia tráfico 3 "Pavimento tránsito peatonal moderado" [17], lo que indica que aunque el volumen removido de las muestras en el análisis de la abrasión profunda haya sido superior, las características de uso del producto se podrían mejorar y por ende su precio comercial en el mercado regional, nacional e incluso internacional y adicionalmente podría pensarse en la fabricación de otros productos que exijan estas propiedades.

3.4.2 Análisis comparativo de los resultados obtenidos entre el proceso de cocción de M2 realizado en horno colmena con atmosfera reductora a temperatura entre $1030^{\circ} \mathrm{C}-1080^{\circ} \mathrm{C}$ (1 hora y 30 minutos de reducción) y la curva de cocción propuesta en el horno mufla.
Enero - Junio 2015

ISSN 0122-820X

PP: 84-94 
Materia prima para la industria cerámica de Norte de Santander. II. Evaluación del comportamiento térmico y su incidencia en las propiedades tecnológicas

Tabla IV. Tabla comparativa entre los hornos mufla y colmena reductora alta.

\begin{tabular}{|c|c|c|c|c|}
\hline \multirow{3}{*}{ HORNO } & NTC 4321-3 & $\begin{array}{c}\text { ANÁLISIS } \\
\text { FÍSICO-CERÁMICO ASTM } \\
\text { C326-03 } \\
\end{array}$ & NTC 4321-4 & NTC 4321-6 \\
\hline & $\begin{array}{c}\text { ABSORCIÓN DE } \\
\text { AGUA }\end{array}$ & CONTRACCIÓN COCIDA & $\begin{array}{c}\text { RESISTENCIA A } \\
\text { LA FLEXIÓN O } \\
\text { MODULO DE } \\
\text { RUPTURA } \\
\end{array}$ & $\begin{array}{c}\text { RESISTENCIA A LA } \\
\text { ABRASIÓN PROFUNDA }\end{array}$ \\
\hline & $\%$ & $\%$ & $\mathbf{N} / \mathbf{m m}^{2}$ & $\mathbf{m m}^{3}$ \\
\hline MUFLA & 7,13 & 3,05 & 25,55 & 165 \\
\hline $\begin{array}{l}\text { COLMENA ATMOSFERA } \\
\text { REDUCTORA ALTA (M2) }\end{array}$ & 8,52 & 2,77 & 16,68 & 184 \\
\hline
\end{tabular}

Fuente: Autores

Realizando un análisis comparativo con los resultados obtenidos en el proceso de cocción, se puede determinar; que con las características de resistencia a la flexión y la abrasión profunda, el producto del horno industrial se clasifica en la referencia tráfico 1 "Paramento", mientras que el obtenido usando la curva de cocción propuesta es de tráfico 2 "Pavimento tránsito peatonal leve" [17], lo que indica que se mejora considerablemente las características del producto; y de igual su precio comercial del material.

3.4.3. Análisis comparativo de los resultados obtenidos entre el proceso de cocción de M3 realizado en horno colmena con atmosfera oxidante a temperatura entre $1050^{\circ} \mathrm{C}-1100^{\circ} \mathrm{C}$ y la curva de cocción propuesta en el horno mufla.

Tabla V. Tabla comparativa entre los hornos mufla y colmena oxidante.

\begin{tabular}{|c|c|c|c|c|}
\hline \multirow{3}{*}{ HORNO } & NTC 4321-3 & $\begin{array}{c}\text { ANÁLISIS } \\
\text { FíSICO-CERÁMICO ASTM } \\
\text { C326-03 }\end{array}$ & NTC 4321-4 & NTC 4321-6 \\
\hline & $\begin{array}{c}\text { ABSORCIÓN DE } \\
\text { AGUA }\end{array}$ & CONTRACCIÓN COCIDA & $\begin{array}{c}\text { RESISTENCIA A } \\
\text { LA FLEXIÓN O MODULO } \\
\text { DE RUPTURA }\end{array}$ & $\begin{array}{c}\text { RESISTENCIA A LA } \\
\text { ABRASIÓN } \\
\text { PROFUNDA } \\
\end{array}$ \\
\hline & $\%$ & $\%$ & $\mathbf{N} / \mathbf{m m}^{2}$ & $\mathbf{m m}^{3}$ \\
\hline MUFLA & 6,96 & 3,49 & 26,80 & 139 \\
\hline $\begin{array}{c}\text { COLMENA } \\
\text { ATMOSFERA } \\
\text { OXIDANTE (M3) }\end{array}$ & 7,59 & 3,60 & 25,87 & 174 \\
\hline
\end{tabular}

Fuente: Autores

Realizando un análisis comparativo de los resultados obtenidos en el proceso de cocción realizado en el horno colmena con atmosfera oxidante a temperatura entre $1050^{\circ} \mathrm{C}-1100^{\circ} \mathrm{C}$, con el proceso realizado en el horno mufla, se puede determinar; que ambos productos obtenidos se clasifican en la referencia tráfico 2 "Pavimento tránsito peatonal leve" [17]. Sin embargo, debe considerarse que en el análisis de resistencia a la abrasión profunda, el volumen removido en el material sinterizado en el horno mufla con la curva propuesta es menor, los que mejora considerablemente las características del producto, y de igual manera el precio comercial de este.

\section{Conclusiones}

En los estudios térmicos se observó que el material debe ingresar a cocción completamente seco, y evitar la rehidratación característica de arcillas illiticas, así disminuir defectos como fisuras o desconchados; además de lograr una buena sinterización pues el material empieza a generarla a partir de temperaturas entre $1000^{\circ} \mathrm{C}$ a $1200^{\circ} \mathrm{C}$. 
La aplicación de la curva de cocción propuesta mejoró las propiedades tecnológicas como la absorción de agua, disminuyendo 3,78\% para M1; $16,31 \%$ para M2 y $8,21 \%$ para M3, con respecto a las absorciones arrojadas en las curvas usadas en los hornos industriales; lo que genera un aumento del valor comercial en un producto tanto a nivel regional, nacional e incluso internacional.

La curva propuesta mejoró los parámetros de uso del material incrementando el porcentaje de resistencia mecánica en $0,12 \%$ para $\mathrm{M} 1 ; 53,18 \%$ para $\mathrm{M} 2,3,59 \%$ para $\mathrm{M} 3$, en referencia a los resultados resistencia en los hornos industriales. Y disminuyó en 10,33\% y $20,11 \%$ para M2 y M3 respectivamente, en referencia al volumen removido en la abrasión profunda.

Los mecanismos de sinterización del material antes de llegar a las temperaturas finales, fueron más eficaces durante el proceso de cocción, esto se comprueba con la mejora en las propiedades tecnológicas, bajando a $6,96 \%$ la absorción de agua y aumentando el umbral por encima de $25 \mathrm{~N} / \mathrm{mm}^{2}$ en referencia a resistencia mecánica.

En el caso de fabricar baldosas cerámica se amplió el tráfico para M1 de nivel 2 a nivel 3 , para en M2 de nivel 1 a nivel 2 y para M3 aunque no se amplió el tráfico; se mejoraron las propiedades de resistencia a la flexión y de abrasión profunda en un valor de 3,59\% y de $20,11 \%$ respectivamente. Es decir, los resultados de la curva de cocción propuesta a nivel de laboratorio, predicen que los productos obtenidos serán de mayor calidad y con la posibilidad de incursionar a mercados de un tráfico superior al tradicional.

\section{Agradecimientos}

Los autores expresan su agradecimiento al Centro de Investigación de Materiales Cerámicos CIMAC de la Universidad Francisco de Paula Santander de CúcutaColombia por el apoyo brindado para la realización de los ensayos, igualmente a la $\mathrm{PhD}$. en ciencias-química Diana Alexandra Torres Sánchez de la Universidad de Pamplona-Colombia, por la colaboración prestada en la realización de los análisis térmicos; a la tecnóloga Claudia Cortes de Ladrillera Casablanca y al tecnólogo químico Carlos Pedraza de Tejar De Pescadero, de Cúcuta-Colombia, por la ayuda en la cocción del material en los hornos industriales.

\section{Referencias}

[1] L. Esposito y A. Tucci, "Abrasión de superficies de azulejos cerámicos esmaltados", Bol. Soc. Esp. Ceram. Vidr., vol. 39, no. 1, pp. 165-171, 2000.

[2] A. Abitante, C. Bergmann y J. Ribeiro, "Abrasión en baldosas cerámicas esmaltadas: Consideraciones sobre la vida útily evaluación de su desempeño", Engenharia Civil, no. 29, pp. 71-79, 2007.

[3] A. M. Bernardin, D. Medeiros, H. G. Calatzis da Silva y H. Gracher Riella, "La piroplasticidad del gres porcelánico", Qualicer, pp. 207-213, 2006.

[4] F. Gene, D. Minichelli y M. G. Parasporo, "Cocción de baldosas cerámicas rústicas de pasta roja en hornos de rodillo mediante radicación", Qualicer, pp. 3-6, 1998.

[5] M. Fernández Abajo. El moldeo por extrusión de los productos, en Manual sobre fabricación de baldosas, tejas y ladrillos, Igualada: Laboratorio Técnico Cerámico S.L. p. 375, 2000.

[6] V. Aleixandre, J. M. González y F. Sandoval, "Estudio fisicoquímico y tecnológico de un grupo de arcillas para ladrillería", Bol. Soc. Esp. Ceram.
Enero - Junio 2015

ISSN 0122-820X

PP: 84-94 
J. D. Santos, P. Y. Malagón y E. M. Córdoba, "Caracterización de arcillas y preparación de pastas cerámicas para la fabricación de tejas y ladrillos en la región de Barichara, Santander", Dyna, vol. 78, no. 167, pp. 53-61, Junio 2011.

C. Vieira, R. Sánchez y S. N. Monteiro, "Characteristics of clays and properties of building ceramics in the state of Rio de Janeiro, Brazil", Construction and Building Materials, vol. 22, no. 5, p. 781-787, Mayo 2008.

[9] A. Escardino, J. E. Enrique y J. L. Amores, "Estudio de pastas de gres para pavimentos", Bol. Soc. Esp. Ceram. Vidr., vol. 20, no. 1, pp. 17-24, Marzo-Abril 1981.

[10] R. A. Muñoz, J. A. Muñoz, P. Mancilla y J. E. Rodríguez, "Caracterización fisicoquímica de arcillas del municipio de guapi - costa pacífica caucana (Colombia)", Rev. Acad. Colomb. Cienc., vol. 31, no. 121, pp. 537-544, Diciembre 2007.

[11] A. Escardino, J. E. Enrique y E. Ramos, "Arcillas cerámicas de la región valenciana. Estudio de algunas arcillas empleadas en la fabricación de azulejos en las provincias de Castellón y Valencia", Bol. Soc. Esp. Ceram. Vidr., vol. 17, no. 2, pp. 83-90, 1977.

[12] SACMI Iberica, S.Ay ATC - Asociación Española de Técnicos Cerámicos, Tecnología cerámica aplicada, vol. II, Castellón: Faenza Editrice Ibérica, S.L. p. 432, 2004.

[13] V. Sánchez Sánchez, "Proyecto para la construcción de un horno cerámico de combustión mixta: gas propano y combustibles vegetales", Proyecto Final de Carrera en la Escuela Superior de Cerámica de Manises. Manises, Valencia, España, 2013.

[14] V.-G. Lee y T.-H. Yeh, "Sintering effects on the development of mechanical properties of fired clay ceramics", Materials Science and Engineering, vol. A, no. 485, pp. 5-13, 2008.

[15] F. Sandoval y A. Ibañez, "Discusión sobre la influencia de la porosidad en la resistencia mecánica de las baldosas cerámicas", Bol. Soc. Esp. Ceram. Vidr., vol. 39, no. 2, pp. 255-258, 2000.

[16] S. Bueno y A. A. Illana. Caracterización de la arcilla gris de de Almuradiel, en Estudio de caracterización tecnológica de la arcilla gris de Almuradiel, Bailen: Centro Tecnologico Innovarcilla, p. 32, 2010.

[17] J. R. García y A. Peñín. Clasificación de baldosas cerámicas según su uso, en Guía de la baldosa cerámica, Valencia: Asociación Española de Fabricantes de Azulejos, Pavimentos y Baldosas Cerámicas -ASCER-. p. 106, 2012.

[18] A. Escardino, M. C. Gastoldo y J. L. Amorós, "Arcillas cerámicas del país Valenciano. IV Estudio de algunas arcillas del Maestrazgo", Bol. Soc. Esp. Ceram. Vidr., vol. 19, no. 5, pp. 341345, 1980.

[19] L. Cely Illera, "Comportamiento térmico y mecánico de una arcilla de la región de Norte de Santander", Tesis de Maestría en la Universidad Francisco de Paula Santander -UFPS-, Cúcuta, Colombia, 2014. 